\title{
Actualidades en el tratamiento nutricional de pacientes con galactosemia clásica
}

\section{Current treatment of classic galactosemia}

Lizbeth López-Mejía, ${ }^{1}$ Mónica Vergara-Vázquez, ${ }^{2}$ Fabiola López-Olivan, ${ }^{2}$ Sara Guillén-López ${ }^{1}$

\begin{abstract}
Resumen
La galactosemia clásica es un error innato del metabolismo de los hidratos de carbono. Existe evidencia reciente que iniciar el tratamiento de forma temprana puede revertir, exitosamente, las complicaciones de esta enfermedad. El tratamiento nutricional cambió en los últimos 20 años; en la actualidad consiste en una dieta restringida de por vida en las principales fuentes de galactosa, como los lácteos y sus derivados, vísceras y productos de soya fermentada. Entre los alimentos permitidos están todos los tipos de frutas, verduras, leguminosas (incluidos los productos con soya no fermentada), oleaginosas, productos de origen animal, aditivos como caseinato de sodio y calcio. La medición de las concentraciones de calcio y vitamina D en plasma es necesaria como parte del seguimiento de estos pacientes. La educación nutricional a pacientes y familiares es importante para mantener las restricciones dietéticas de por vida.
\end{abstract}

PALABRAS CLAVE: Galactosemia clásica; metabolismo de los hidratos de carbono; galactosa-1 fosfato; galactosemias; metabolismo de carbohidratos.

\section{Abstract}

Classical galactosemia is an inborn error of carbohydrate metabolism, recent evidence suggests that starting early diet treatment can successfully reverse complications of this disease. Nutritional treatment has changed over the last 20 years, currently a galactose restricted diet is recommended for a lifetime, the main sources of galactose such as milk and dairy products, as well as organ meats, meat by products and fermented soy products are not allowed. Permitted foods are all kinds of fruits, vegetables, legumes (including products based on non-fermented soybeans), oilseeds, animal products, and additives such as sodium and calcium caseinate. Calcium and vitamin D must be monitored as part of the follow-up. Nutritional education is important to patients and their families in order to maintain a lifetime diet restriction.

KEYWORDS: Classical galactosemia; Carbohydrate metabolism; Galactose-1 phosphate; Galactosemias; Carbohydrate Metabolism.

\begin{abstract}
${ }^{1}$ Laboratorio de Errores Innatos del Metabolismo y Tamiz, Instituto Nacional de Pediatría, Secretaría de Salud, Ciudad de México.

${ }^{2}$ Dirección de Atención a la Salud Materna y Perinatal, Centro Nacional de Equidad de Género y Salud Reproductiva, Secretaría de Salud, Ciudad de México.

Correspondencia

Sara Guillén López

sara_guillen@hotmail.com

Este artículo debe citarse como López Mejía L, Vergara Vázquez M,López Olivan F, Guillén López S. Actualidades en el tratamiento nutricional de la galactosemia clásica.Acta Pediatr Mex. 2018;SI(39):75S-80S.
\end{abstract}

\section{INTRODUCCIÓN}

A partir de 2011, la detección oportuna de la galactosemia se incorporó al panel obligatorio de tamiz neonatal de la Secretaría de Salud ${ }^{1}$ y los fundamentos del tratamiento se dieron a conocer en el Lineamiento Técnico para la Detección, Diagnóstico, Tratamiento y Seguimiento de los Errores Innatos del Metabolismo emitido por la Secretaría de Salud en el año 2010. ${ }^{2}$ La 
investigación en salud es por demás dinámica y los avances exigen que los profesionales de este ramo permanezcan siempre actualizados. Por eso el objetivo de esta revisión consiste en resaltar las últimas tendencias en el tratamiento nutricional de los pacientes con galactosemia.

La galactosemia clásica es una enfermedad genética, de herencia autosómica recesiva, en la que existe una alteración en la producción de la enzima galactosa-1-fosfato uridiltransferasa (GALT). Esta enzima interviene en el metabolismo de la galactosa-1-fosfato y UDP-glucosa a UDP-galactosa y glucosa-1-fosfato. ${ }^{3}$ La galactosa es una aldohexosa con funciones metabólicas y estructurales porque forma parte de varias macromoléculas, como gluco-conjugados que incluyen glucoproteínas, glucolípidos y glucosaminoglicanos. Existe una producción endógena importante a partir de estos compuestos; en adultos con galactosemia la síntesis de galactosa puede llegar hasta un gramo por día, pero en niños y lactantes es mayor. Esta producción se ha descrito como un factor importante en la aparición de complicaciones a largo plazo que se observan en individuos adultos. ${ }^{4}$

En 2017 se publicó una guía internacional para el diagnóstico y tratamiento a largo plazo de pacientes con galactosemia clásica en la que se establece que cuando la actividad enzimática es menor al $10 \%$, o ante una variante patogénica en ambos alelos del gen GALT, debe implementarse el tratamiento nutricional. ${ }^{5}$

Ante la evidencia del daño que causa la galactosemia y ante cualquier sospecha clínica, es indispensable iniciar el tratamiento nutricional, inclusive antes de la confirmación diagnóstica. El tratamiento nutricional se basa en una dieta restringida en galactosa; cuando se proporciona tempranamente los síntomas: cataratas, ictericia y anormalidades en la función renal pueden desaparecer de inmediato o en el trascurso de los días. ${ }^{3}$ La fuente primaria en la dieta de galactosa es la lactosa que se encuentra en los productos lácteos: $100 \mathrm{~mL}$ de leche de vaca contienen, aproximadamente, 2,400 mg de galactosa, por lo que todos los lácteos se eliminan en este tratamiento. ${ }^{6}$

\section{Alimentación en los primeros seis meses}

En el caso de lactantes debe suspender la lactancia materna y eliminar de la dieta las fórmulas que contengan lactosa. Se debe iniciar con fórmula de proteína aislada de soya o fórmula muy baja o sin hidratos de carbono que puedan contener galactosa. Si bien la fórmula de soya contiene pequeñas cantidades de galactosa ligada e isoflavonas, éstas no han demostrado tener un efecto clínico adverso y ha sido una de las fórmulas más estudiadas para el tratamiento de esta enfermedad.?

La fórmula de soya no se recomienda en neonatos prematuros porque existen estudios que encontraron bajas concentraciones de fósforo sérico y altas de fosfatasa alcalina, esto deriva en incremento en la incidencia de osteopenia en prematuros alimentados con esta fórmula, en comparación con quienes son alimentados con fórmula derivada de leche de vaca; por lo tanto, para este grupo de pacientes la recomendación es una fórmula elemental. ${ }^{8}$

Las fórmulas elementales de aminoácidos libres no contienen lactosa ni galactosa $y$, si bien la bibliografía no ha reportado efectos secundarios, son pocas las publicaciones que describen su consumo. Zlautunich y su grupo reportaron, en un paciente, una reducción muy rápida de las concentraciones de galactosa-1-fosfato. Se desconoce si este decremento tiene relación con el pronóstico a largo plazo porque no existen, a la fecha, estudios prospectivos con una muestra significativa que permita emitir una recomendación sólida. ${ }^{9,10}$ 


\section{Alimentación complementaria en el paciente con galactosemia}

La introducción de alimentos sólidos debe iniciarse alrededor de los seis meses de edad, cuando existe una adecuada maduración de la función renal y gastrointestinal para metabolizar alimentos distintos a la fórmula. Es necesario que los niños tengan las habilidades motoras adecuadas para ingerir alimentos de forma segura. Los hitos del desarrollo que deben mostrar para iniciar la alimentación complementaria son: adecuado control cefálico, sentarse sin apoyo, desaparición del reflejo de extrusión, llevarse las manos a la boca y utilizar la lengua para Ilevar los alimentos a la parte posterior de la boca. ${ }^{11}$

Durante el decenio de 1990 surgió evidencia que indicaba que existe un contenido importante de galactosa libre, oligómeros de galactosa, galactosa ligada a proteína, lípidos y galactosa en la pared celular en frutas y verduras, leguminosas. ${ }^{12,13}$ Por eso, durante varios años se indicó una restricción importante en la dieta de los pacientes en varios países. En 2014 Van Calcar y su grupo revisaron el contenido de estos alimentos y concluyeron que la restricción de frutas, verduras y leguminosas no contribuye al aumento de las concentraciones eritrocitarias de galactosa 1 fosfato en los pacientes. La mayor parte del contenido de galactosa no está disponible para su digestión en el aparato gastrointestinal, la cantidad que pueden contener es muy pequeña en comparación con la producción endógena; esta restricción implica la disminución en el aporte de fibra, vitamina A, C, K, compuestos antioxidantes, fitoquímicos, entre otros. ${ }^{14}$

En otro estudio de Van Calcar y coautores se reevaluaron las concentraciones de galactosa en diferentes leguminosas y encontraron concentraciones inferiores a las reportadas previamente. ${ }^{15}$ Aunado a lo anterior se encontró que existía una variación en el contenido de galactosa depen- diendo de la región de donde provenían las frutas y verduras, su maduración, exposición a algún proceso térmico, su forma de almacenamiento, entre otras. Debido a todos estos factores esto hace poco práctica cualquier recomendación porque no se considera parte de la terapia nutricional, la restricción de alguna fruta, verdura o leguminosa. 5,14

En la dieta del paciente con galactosemia deben restringirse el consumo de productos lácteos y sus derivados porque estos proporcionan una fuente importante de calcio y vitamina D. Al eliminarse de la dieta debe iniciarse una suplementación adecuada y evaluar cada año las concentraciones plasmáticas de la 1,25-dihidroxi vitamina D, y la masa ósea con la densitometría o DEXA a partir de los 8 a 10 años. La vigilancia estrecha es indispensable para poder proporcionar los requerimientos de la ingestión diaria recomendada de calcio y vitamina D. ${ }^{5,16}$

\section{Alimentación en niños y adolescentes}

Existe evidencia que señala que con la edad, la tolerancia de galactosa se incrementa. Esto parece ser diferente para cada individuo porque se ha observado que no todos los pacientes con galactosemia tienen complicaciones, aún a pesar de tener una de las mutaciones que se han descrito como más graves, que es la Q188R.17,18 En esta etapa puede ampliarse un poco más la variedad de alimentos en pacientes con evolución clínica y bioquímica favorable.

Antes solo se recomendaba la administración de fórmulas con aislados de proteína de soya como sustituto a las fórmulas de leche de vaca; sin embargo, en la actualidad está indicada cualquier bebida a base de soya. Si bien se les agrega carragenina como emulsificador y éste contiene enlaces de galactosa b 1-4 y a 1-3, 3,6 anhidro galactosa y sulfato de galactosa, estos no están disponibles para la digestión en el tubo gastrointestinal de los humanos. ${ }^{14}$ 
La Guía Internacional para el Diagnóstico y Tratamiento a largo plazo de los pacientes con galactosemia clásica recomienda que se permita la ingestión de quesos maduros cuyo contenido de galactosa sea menor a $25 \mathrm{mg}$ por cada 100 gramos. ${ }^{5}$ Existen análisis detallados del contenido de quesos maduros Ilevados a cabo en Europa ${ }^{19,20}$ y Estados Unidos ${ }^{15}$ que reportan que los quesos artesanales, a diferencia de los industrializados, tienen menor cantidad de lactosa. El contenido puede variar dependiendo de su procesamiento y empaque. En el caso de México aún no se ha efectuado una cuantificación bromatológica de productos nacionales que permita emitir una recomendación. Solo se podrían llegar a recomendar los quesos importados con contenido de galactosa descrito. ${ }^{21}$
Por lo que se refiere a los caseinatos que antes se restringían, se sabe que el proceso para aislar proteínas de la caseína y solubilizarlas para formar caseinatos hace que la cantidad de lactosa y, por tanto, de galactosa sean mínimas en el producto: en promedio, $35 \mathrm{mg} / 100 \mathrm{~g}$. Estos caseinatos se usan en pequeñas cantidades en alimentos para niños, embutidos y panadería y pueden incluirse en la dieta del paciente. Continúa la restricción de ingredientes derivados de la leche: suero, caseína, proteína de leche en polvo, sólidos de leche, proteína de suero hidrolizada, proteína hidrolizada de caseína, lactosa, lactoalbúmina y suero. ${ }^{5,14}$ En el Cuadro 1 se resumen las recomendaciones de acuerdo con la edad para pacientes con galactosemia. $., 6,14,15$

Cuadro 1. Recomendaciones de los diferentes grupos de alimentos para los pacientes con galactosemia, por grupos de edad

\begin{tabular}{|c|c|c|c|c|c|c|c|}
\hline Edad & Fórmula & Fruta & Verdura & Cereales & $\begin{array}{l}\text { Productos de } \\
\text { origen animal }\end{array}$ & Grasa & Leguminosas \\
\hline 0 a 6 meses & $\begin{array}{l}\text { - Fórmula } \\
\text { sin o con } \\
\text { mínimas } \\
\text { cantidades } \\
\text { de galactosa } \\
\text { como fórmula } \\
\text { de soya }\end{array}$ & - & - & - & - & - & - \\
\hline 6 meses a 1 año & $\begin{array}{l}\text { - Fórmula } \\
\text { sin o con } \\
\text { mínimas } \\
\text { cantidades } \\
\text { de galactosa }\end{array}$ & Todas & Todas & $\begin{array}{l}\text { Todos } \\
\text { - Arroz, maíz, trigo, } \\
\text { avena, quinoa, } \\
\text { amaranto. } \\
\text { Preferir naturales. } \\
\text { En caso de dar } \\
\text { industrializado } \\
\text { checar etiquetas y } \\
\text { evitar: caseína, sue- } \\
\text { ro, lactoalbúmina, } \\
\text { lactosa, proteína de } \\
\text { leche hidrolizada }\end{array}$ & $\begin{array}{l}\text { Permitidos: } \\
\text { Res, cerdo, } \\
\text { pollo, pesca- } \\
\text { do, huevo. } \\
\text { Prohibidos: } \\
\text { - Vísceras } \\
\text { como hígado, } \\
\text { riñón, cora- } \\
\text { zón. } \\
\text { - Quesos }\end{array}$ & $\begin{array}{l}\text { Todas excepto } \\
\text { mantequilla o } \\
\text { que conten- } \\
\text { gan algún } \\
\text { ingrediente } \\
\text { prohibido de } \\
\text { la leche }\end{array}$ & $\begin{array}{l}\text { Todas } \\
\text { - Frijoles, lente- } \\
\text { jas, garbanzos, } \\
\text { haba, soya sin } \\
\text { fermentar. }\end{array}$ \\
\hline $\begin{array}{l}\text { Niños y adoles- } \\
\text { centes }\end{array}$ & $\begin{array}{l}\text { Fórmula sin o } \\
\text { con mínimas } \\
\text { cantidades de } \\
\text { galactosa } \\
\text { - Fórmula } \\
\text { y bebidas a } \\
\text { base de soya }\end{array}$ & Todas & Todas & $\begin{array}{l}\text { Todos } \\
\text { - Arroz, maíz, trigo, } \\
\text { avena, quinoa, } \\
\text { amaranto. } \\
\text { Preferir naturales. } \\
\text { En caso de dar } \\
\text { industrializado } \\
\text { checar etiquetas y } \\
\text { evitar: caseína, sue- } \\
\text { ro, lactoalbúmina, } \\
\text { lactosa, proteína de } \\
\text { leche hidrolizada }\end{array}$ & $\begin{array}{l}\text { Permitidos: } \\
\text { Res, cerdo, } \\
\text { pollo, pesca- } \\
\text { do, huevo. } \\
\text { Prohibidos: } \\
\text { - Vísceras } \\
\text { como hígado, } \\
\text { riñón, cora- } \\
\text { zón. } \\
\text { - Quesos }\end{array}$ & $\begin{array}{l}\text { Todas excepto } \\
\text { mantequilla o } \\
\text { que conten- } \\
\text { gan algún } \\
\text { ingrediente } \\
\text { prohibido de } \\
\text { la leche }\end{array}$ & $\begin{array}{l}\text { Todas } \\
\text { - Frijoles, lente- } \\
\text { jas, garbanzos, } \\
\text { haba, soya. Sin } \\
\text { fermentar }\end{array}$ \\
\hline
\end{tabular}


Importancia de la lectura correcta del etiquetado de los alimentos

Los padres, y a su debido tiempo los pacientes, deben estar capacitados para interpretar correctamente la información de las etiquetas, no sólo de alimentos como la leche en polvo, sólidos de leche, proteína de suero hidrolizada, sino también de otros productos como: medicamentos, pastas dentales, entre otros. El objetivo principal de proveer información a la familia es el de identificar posibles fuentes de galactosa porque los ingredientes de los productos pueden cambiar sin previo aviso, ${ }^{3}$ lo que hace necesaria la revisión constante de las etiquetas. Las vísceras de animales, productos de soya fermentados como: miso, natto, tempeh, sufu, salsa de soya fermentada pueden agregarse o usarse como condimentos en algunos alimentos industrializados o como parte de la cocina asiática. ${ }^{14}$ Mantener al paciente informado, y a su familia o cuidadores, permitirá tener un adecuado control metabólico y prevenir complicaciones.

\section{CONCLUSIÓN}

Las evidencias más recientes señalan que la dieta de los pacientes con galactosemia puede ser más amplia porque ahora incluye todas las frutas, verduras, oleaginosas y leguminosas. Esto es importante porque además de dar mayor calidad de vida a los pacientes, se reduce el riesgo de deficiencias nutricionales asociadas con las severas restricciones de las recomendaciones anteriores. Sigue siendo relevante vigilar la nutrición del paciente para evitar la elevación de las concentraciones de galactosa y prevenir deficiencias de calcio y vitamina D.

\section{REFERENCIAS}

1. Norma Oficial Mexicana NOM-034-SSA2-2013, Para la prevención y control de los defectos al nacimiento. Diario Oficial de la Federación. México 2014. [Citado 8 septiembre 2018]. Disponible en: https://www.gob.mx/
salud\%7Ccnegsr/documentos/norma-oficial-mexicananom-034-ssa2-2013-para-la-prevencion-y-control-de-losdefectos-al-nacimiento-118923

2. Centro Nacional de Equidad de Género y Salud Reproductiva. Lineamiento Técnico de Tamiz Neonatal, Detección, Diagnóstico, Tratamiento y Seguimiento de los Errores Innatos del Metabolismo. 2010.

3. Berry GT, Walter JH. Disorders of galactose metabolism. In: Saudubray JM, van den Berghe G, Walter JH, eds. Inborn Metabolic Diseases - Diagnosis and Treatment. 5th ed. New York: Springer Inc.; 2012:140-149.

4. Berry GT, Moate PJ, Reynolds RA, Yager CT, Ning C, Boston $\mathrm{RC}$, et al. The rate of the novo galactose synthesis in patients with galactose-1-phosphate uridyltransferase deficiency. Mol Genet Metab. 2004; 81(1): 22-30.

5. Welling L, Bernstein L, Berry G, Burlina A, Eyskens F, Gautschi $M$, et al. International clinical guideline for the management of classical galactosemia: diagnosis, treatment, and follow-up. J Inherit Metab Dis. 2017; 40 (2): 171-176.

6. Bernstein L, Van Calcar S. The diet for Galactosemia in: Bernstein L, Helm J, Rohr F. Nutrition Management of Inherited metabolic diseases. Springer. 2015, USA. pp. 285- 293.

7. Merritt RJ, Jenks BH. Safety of soy-based infant formulas containing isoflavones: the clinical evidence. J Nutr. 2004; 134(5):1220S- 1224S.

8. Bhatia J, Greer F. Committee on Nutrition, American Academy of Pediatrics. Use of soy protein-based formulas in infant feeding. Pediatrics. 2008; 121 (5): 1062-1068.

9. Zlautunich CO, Packman S. Galactosemia: early treatment with an elemental formula. J Inherit Metab Dis. 2005; 28(2):163-8.

10. Ficicioglu C, Hussa C, Yager C, Segal S. Effect of galactose free formula on galactose-1-phosphate in two infants with classical galactosemia. Eur J Pediatr. 2008; 167(5): 595-6.

11. Fewtrell M, Bronsky J, Campoy C, Domellöf M, Embleton N, Fidler Mis N, Hojsak I, Hulst JM, Indrio F, Lapillonne A, Molgaard C. Complementary Feeding: A Position Paper by the European Society for Paediatric Gastroenterology, Hepatology, and Nutrition (ESPGHAN) Committee on Nutrition. J Pediatr Gastroenterol Nutr. 2017; 64 (1): 119-132.

12. Gross K, Acosta PB. Fruits and vegetables are a source of galactose: implications in planning the diets of patients with galactosemia. J Inherit Metab Dis. 1991; 14(2): 253-258.

13. Gropper S, Weese J, West P, Gross KC. Free galactose content of fresh fruits and strained fruit and vegetable baby foods: more foods to consider for the galactose restricted diet. J Am Diet Assoc. 2000; 100 (5): 573-575.

14. Van Calcar S, Bernstein L, Rohr F, Scaman CH, Yannicelli $\mathrm{S}$, Berry GT. A re-evaluation of life-long severe galactose restriction for the nutrition management of classic galactosemia. Mol Genet Metab. 2014; 112 (3):191-197. 
15. Van Calcar SC, Bernstein LE, Rohr FJ, Yannicelli S, Berry GT, Scaman $\mathrm{CH}$. Galactose content of legumes, caseinates, and some hard cheeses: implications for diet treatment of classical galactosemia. J Agric Food Chem. 2014; 62 (6): 1397-402.

16. Panis $B$, Vermeer $C$, van Kroonenburgh MJPG, Nieman FHM, Menheere PPCA, Spaapen LJ, et al. Effect of calcium, vitamins $\mathrm{K} 1$ and D3 on bone in galactosemia. Bone. 2006; 39(5): 1123-1129.

17. Berry GT, Elsas LJ. Introduction to the Maastricht workshop: lessons from the past and new directions in galactosemia. J Inherit Metab Dis. 2011; 34 (2): 249-255.
18. Panis B. Bakker JA, Sels JP, Spaapen LJ, van Loon LJ, Rubio-Gozalbo ME. Untreated classical galactosemia with mild phenotype. Mol Genet Metab. 2006; 89(3): 277-279.

19. Portnoi PA, MacDonlad A. The lactose and galactose content of cheese suitable for Galactosemia: New Analysis. JIMD Rep. 2016; 29: 85-87.

20. Portnoi PA, MacDonald A. Determination of the lactose and galactose content of cheese for use in the galactosemia diet. J Hum Nutr Diet. 2009; 22 (5):400-8.

21. Portnoi PA, Macdonald A. The lactose content of Mini Babybel and suitability for Galactosemia. J Hum Nutr Diet. 2011; 24(6): 620-1. 